Service social

\title{
L'organisation communautaire : définition et paradigme
}

\section{Gérald Doré}

Volume 34, numéro 2-3, 1985

L'organisation communautaire

URI : https://id.erudit.org/iderudit/706269ar

DOI : https://doi.org/10.7202/706269ar

Aller au sommaire du numéro

Éditeur(s)

École de service social de l'Université Laval

ISSN

1708-1734 (numérique)

Découvrir la revue

Citer cet article

Doré, G. (1985). L'organisation communautaire : définition et paradigme.

Service social, 34(2-3), 210-230. https://doi.org/10.7202/706269ar d'utilisation que vous pouvez consulter en ligne.

https://apropos.erudit.org/fr/usagers/politique-dutilisation/ 
DORÉ, Gérald, professeur à l'École de service social de l'Université Laval.

\section{L'organisation communautaire : définition et paradigme}

\section{Gérald Doré}

Cet article systématise un contenu élaboré au fil de mes onze années d'enseignement de l'organisation communautaire à l'École de service social de l'Université Laval, avec la collaboration de plusieurs collègues dont certains sont encore dans l'enseignement, à Laval ou ailleurs, et d'autres retournés dans la pratique. Je pense aux Denis Fortin, Yves Hurtubise, Jocelyne Barnabé, Denis Plamondon, Vicente de Paula Faleiros et André Jacob avec qui j'ai, soit donné le cours Organisation communautaire $\mathrm{I}$, soit préparé ou enrichi le contenu à travers des discussions.

À l'hiver 1983, dernier trimestre où j'ai donné ce cours, j'ai nettement pris conscience de l'utilité d'en codifier les principaux éléments pour une diffusion plus large dans les milieux intéressés à l'organisation communautaire.

Le sentiment d'urgence à cet égard m'est venu de la constatation triviale que la tradition orale de l'enseignement et des échanges entre intervenants ne suffit plus, à l'heure actuelle, à faire circuler les acquis de base en organisation communautaire, au Québec. L'heure est aux écrits de synthèse et je n'ai ici d'autre objectif que d'apporter ma contribution en la matière.

\section{L'organisation communautaire: définition}

La définition de l'organisation communautaire, telle que dite dans les milieux où elle se pratique et telle que conceptualisée dans la littérature qui la commente, comporte trois paliers, du plus général au plus particulier : comme pratique sociale, comme méthode d'intervention en service social, et comme modèle normatif d'intervention. 


\section{Comme pratique sociale}

Au plus haut niveau de généralité, l'organisation communautaire est une pratique sociale. Cette pratique consiste à intervenir dans des collectivités, dans le but de susciter leur mobilisation et leur insertion dans des processus de réalisation de changements sociaux. ${ }^{1}$ Les agents et agentes qui la mettent en ouvre sont à la disposition des communautés locales ou des associations volontaires sectorielles (syndicats, coopératives, groupes populaires, corporations sans but lucratif), dans le but d'aider leurs membres à s'organiser pour prendre collectivement en charge les situations-problèmes auxquels ils sont confrontés. "L'action sociale basée sur la participation active des intéressés " (Salberg et Welsh-Bonnard, 1970: 52) est la marque distinctive qui permet de repérer cette pratique spécifique dans la totalité complexe et multiforme des pratiques sociales. Dans les années soixante-dix, le terme "intervention collective" était fréquemment utilisé dans les écoles et départements de service social et de travail social du Québec pour référer à cette définition générale de l'organisation communautaire.

À ce palier de définition, l'organisation communautaire n'est évidemment pas l'apanage de ceux et celles qui la pratiquent sous le titre d'organisateurs ou d'organisatrices communautaires. Son existence est antérieure à son développement sous forme de spécialisation professionnelle et, aujourd'hui encore, elle se déploie aussi bien au-dehors qu'au-dedans du cadre de la spécialisation professionnelle.

Meister en donne comme exemples (avant la lettre) les innombrables associations créées dès le dix-neuvième siècle durant la période d'industrialisation des pays occidentaux (Meister, 1962: 3). Les précurseurs sont ces milliers d'intervenants et intervenantes anonymes qui, à titre volontaire et bénévole, ont suscité la mobilisation de leurs compagnons et compagnes de travail, de leurs concitoyens et concitoyennes, dans des syndicats, des coopératives, des partis politiques et des associations diverses.

Au Québec comme ailleurs, bien avant d'être intégrée comme méthode d'intervention en service social et comme titre d'emploi dans les établissements publics de services sociaux, l'organisation communautaire a existé dans les syndicats, les coopératives et les mouvements d'action catholique (Harvey, $1978: 291$ ), aussi bien que dans l'éducation agricole (Jean, 1978). Et elle continue de déborder le cadre de la spécialisation professionnelle.

Dans le cadre même de cette spécialisation, cette pratique est réalisée sous de multiples titres d'emploi (Doré et Larose, 1979 : 70-71) et par des agents et agentes dont le groupe de référence significatif est 
souvent beaucoup plus l'organisation avec qui ils et elles travaillent que la communauté professionnelle des organisateurs et organisatrices communautaires, si tant est qu'une telle entité existe. C'est nettement le cas, par exemple, des agents et agentes qui font de l'organisation communautaire, sous le titre de permanents ou permanentes, dans les grandes organisations syndicales.

\section{Comme méthode d'intervention en service social}

Au deuxième palier de définition, l'organisation communautaire est une méthode d'intervention en service social, au même titre que le service social personnel (casework) et le service social des groupes (groupwork).

Paradoxe parmi d'autres, les premières définitions institutionnalisées ne nous renvoient pas à l'univers de contenu du premier palier, tel que nous venons de le décrire, mais bien plutôt à celui connu aujourd'hui sous le terme de gestion des services sociaux.

En 1939, le Lane Report, considéré comme le début de la littérature sur l'organisation communautaire en tant que méthode du service social, lui donne comme champ d'application la création, le développement et la coordination de l'ensemble des services sociaux d'une communauté (Médard, 1969 : 214).

En 1943, la National Association of Social Workers des États-Unis la définit comme une intervention visant à organiser des services et à coordonner les efforts des gouvernements et des agences volontaires de bien-être social (Dunham, 1943: 137). La participation active de ceux et celles vivant les situations-problèmes auxquelles les services sont censés répondre est absente de la définition; ce qui en exclut, à toutes fins pratiques, la différence spécifique qui distingue l'organisation communautaire de la gestion des services sociaux.

Au Québec aussi, c'est associée à un contenu de gestion que la première définition de l'organisation communautaire fait son apparition en service social, au tout début des années soixante. Claude Morin, professeur à l'École de service social de l'Université Laval, l'identifie à l'organisation des "services privés de bien-être" (Morin, 1962). Pierre Laplante, directeur général du Conseil des œuvres de Montréal, utilise le terme pour désigner l'ensemble des activités de son organisme dont "la préoccupation centrale est de voir à ce que la population ait à sa disposition des services de bien-être adéquats" (Laplante, 1962: 110).

Deux expériences, toutes deux commencées en 1963, vont rapidement et fortement contribuer à démarquer, au Québec, l'organisation communautaire naissante de la coordination des oeuvres so- 
ciales: le travail du Conseil des œuvres précité, dans le quartier Saint-Henri, à Montréal, à l'initiative de travailleurs sociaux; et l'intervention du Bureau d'aménagement de l'Est du Québec (B.A.E.Q.) dans le Bas-Saint-Laurent, la Gaspésie et les îles-de-la-Madeleine, à laquelle participent, parmi d'autres professionnels des sciences humaines, des travailleurs sociaux. L'utilisation du terme "animation sociale" au lieu d' "organisation communautaire" vient paradoxalement souligner une appropriation, dans ces deux expériences, du contenu participatif qui caractérise l'organisation communautaire comme pratique sociale distincte. Ce terme aura d'ailleurs tendance à prédominer dans la pratique jusqu'à l'implantation des centres locaux de services communautaires (C.L.S.C.) et l'intégration du titre d'emploi "organisateur communautaire" dans les conventions collectives de ces établissements, dans les années soixante-dix.

Le mouvement qui la démarque de la gestion des services sociaux, au Québec, est en même temps celui qui tend à distendre son lien avec la profession du service social, et en particulier avec sa Corporation issue d'une loi de 1960 de l'Assemblée législative du Québec. Alors que dans Saint-Henri, puis dans d'autres quartiers de Montréal et d'autres villes du Québec, les premiers organisateurs et organisatrices communautaires, sortis des écoles de service social, ${ }^{2}$ s'impliquent avec les populations locales dans des pratiques novatrices qui appellent un renouveau de la profession, la Corporation tend à se replier sur la définition et la défense du statut professionnel des travailleuses sociales et travailleurs sociaux (Carey-Bélanger, 1979). Après avoir alimenté le débat sur la formation en service social à l'intérieur de la Corporation, Michel Blondin (1966), principal définiteur de l'animation sociale en milieu urbain défavorisé, dans les années soixante, en délaisse les activités. Il pose ainsi un geste qui deviendra la règle commune pour la plupart des organisateurs et organisatrices communautaires dans les années qui suivront.

Cette tendance à la distanciation entre l'organisation communautaire et la profession du service social sera accentuée, dans les années soixante-dix, par l'élimination presque complète des rares postes d'organisateurs et organisatrices communautaires dans les agences de service social devenues centres de services sociaux (C.S.S.). La relève est graduellement assumée, il est vrai, par les centres locaux de services communautaires, mais dans un nouveau contexte organisationnel échappant au contrôle de la profession, et où sa reconnaissance institutionnelle comme titre d'emploi est assortie de critères d'embauche qui ne font plus de la formation en service social une obligation pour les détenteurs et détentrices des postes. 
Néanmoins, les liens entre l'organisation communautaire et la profession du service social se maintiennent à travers les programmes de formation des écoles et départements de service social et de travail social; ceux-ci sont les seuls à dispenser une formation spécialisée dans ce champ. Et, malgré l'ouverture des postes à d'autres disciplines, le service social reste encore la discipline de formation du plus grand nombre d'organisateurs et organisatrices communautaires au Québec (Doré et Larose, 1979: 82-83).

\section{Comme modèle normatif d'intervention}

Le troisième palier de définition est plus particulier. II ramène la définition de l'organisation communautaire au modèle normatif de celui qui la pratique. Toute action sociale est basée sur un système de valeurs (Salberg et Welsh-Bonnard, $1976: 61$ ). Toute pratique d'organisation communautaire repose sur une idéologie, une conception de la société, des rapports sociaux, et du changement social en particulier. De cette idéologie, qui peut s'appuyer à des degrés divers sur les théories et les découvertes des sciences sociales contemporaines, découlent des principes d'action ou normes (d'où le terme "normatif") qui tendent à structurer la pratique de l'intervenant ou de l'intervenante. Si celui-ci ou celle-ci systématise, sous une forme transmissible aux autres (écrite ou audio-visuelle), l'idéologie et les principes d'action qui sous-tendent sa pratique, celle-ci est susceptible de devenir un "modèle", c'est-à-dire une pratique codifiée par rapport à laquelle il est possible de se situer, soit pour la répéter, soit pour s'en démarquer, soit pour se définir sa propre pratique.

Le terme "organisation communautaire", dans son sens le plus restreint, est étroitement associé, par exemple, au modèle élaboré dans les années cinquante par Murray G. Ross dans son ouvrage Community Organization : Theory and Principles. Cet ouvrage est sans doute celui qui a le plus contribué, en Amérique du Nord, à distinguer l'organisation communautaire de la gestion, dans la profession du service social. ${ }^{3}$ En partant du principe de la participation de la communauté concernée (Ross, 1967: 40), Ross enracine son modèle dans ce qui, nous l'avons vu, représente la marque distinctive de l'organisation communautaire comme pratique sociale.

Mais, comme la plupart des modèles, celui de Ross tend à se donner comme une représentation de la totalité de la pratique alors qu'il n'en est qu'un des multiples modèles normatifs possibles.

Le besoin, fortement ressenti au fil des années, en rapport avec le développement et la transmission des connaissances en organisation 
communautaire, est précisément celui d'un outil conceptuel qui nous permette de penser la totalité de ses orientations normatives possibles. Le terme " paradigme " $4 \mathrm{~m}$ 'apparaît le plus approprié pour nommer cet outil conceptuel, en ce sens qu'il nous renvoie à un schéma qui représente l'univers des formes possibles d'un phénomène, susceptible de s'actualiser dans une multitude de cas singuliers. De la même façon que la conjugaison du verbe " aimer " peut être dite le "paradigme" de la conjugaison de tous les verbes en "-er" de la langue française, l'outil conceptuel dont nous avons besoin doit, par analogie (la pratique sociale ne se structurant pas comme la langue !), nous permettre de rendre compte de toutes les directions qu'on peut donner à l'action en organisation communautaire et de replacer chacune des pratiques singulières dans la "conjugaison" qui lui correspond.

Les ouvrages généraux qu'il $m$ 'a été donné de consulter au fil des années, me sont toujours apparus incomplets à cet égard. En langue française, par exemple, l'ouvrage, excellent par ailleurs, du Français Jean-François Médard sur l'organisation communautaire aux ÉtatsUnis, ne rend compte que de deux catégories d'orientation normative, qu'il appelle " consensuelle" et " conflictuelle" (Médard, 1969 : 135-185). Le livre des Suisses Salberg et Welsh-Bonnard (1970), qui se présente comme une introduction à l'action communautaire, ne tient compte en réalité que de deux modèles, le développement communautaire des Nations unies et l'organisation communautaire de Ross, tous deux affiliés à la même orientation normative. Le récent ouvrage québécois de Lamoureux, Mayer et Panet-Raymond (1984) s'inscrit explicitement dans les orientations que nous qualifierons plus loin de "pression" et de "politisation" (pp. 7-9 et 27-31). En anglais, l'article classique de Rothman (1970) compare trois modèles sur plusieurs variables significatives avec des exemples appropriés; mais, de l'aveu même de son auteur, il ne vise pas à couvrir tout le champ des modèles possibles (p. 21).

\section{L'organisation communautaire : le paradigme}

Le paradigme proposé ici est le produit d'une double démarche inductive d'utilisation de la littérature sur l'organisation communautaire en tant que pratique sociale, et d'observation d'expériences concrètes. $\mathrm{Au}$ fil de cette démarche, il ressort que la totalité des pratiques concernées peut être pensée sous quatre grandes catégories d'orientation normative, selon la direction que l'on y donne à l'action :

1) Intégration : rend compte des pratiques visant le renforcement de l'intégration des collectivités autour de leaders et de projets reliés aux structures de pouvoir en place ; 
2) Pression : rend compte des pratiques visant la création d'un nouveau rapport de force en faveur des intérêts immédiats de la collectivité exploitée ou dominée à laquelle les intéressés appartiennent ;

3) Appropriation: rend compte des pratiques visant le développement d'entreprises ou de projets économiques contrôlés par ceux et celles qui y travaillent ou qui en consomment les produits ou les services;

4) Politisation: rend compte des pratiques tentant de relier des actions collectives actuelles à des perspectives de transformation des structures politiques.

Ces orientations normatives interagissent avec les dimensions communes à toute pratique d'organisation communautaire, étant influencées par elles (contexte historique, idéologie) ou contribuant à la spécificité de leur contenu (problématique, population-cible, agents d'intervention, processus d'intervention et impact), tel qu'illustré au tableau 1.

À partir d'une étude des traits communs aux expériences de pratique se réalisant dans un même modèle normatif, il serait possible de dégager, dans ce paradigme, le type de pratique qui caractérise les modèles d'intégration, celui qui caractérise les modèles de pression, etc. Il s'agit là d'une piste par où pourrait se poursuivre la conceptualisation à l'intérieur du paradigme de l'organisation communautaire. Depuis quelques années, ce dernier est utilisé dans le premier cours d'organisation communautaire à l'École de service social de l'Université Laval, pour encadrer une démarche pédagogique d'étude, de modèles singuliers de pratique dans les quatre grandes catégories de modèles normatifs.

À titre d'illustration, les tableaux 2, 3, 4 et 5 proposent un chantier d'étude de l'organisation communautaire qui met en valeur trois modèles singuliers dans chaque catégorie de modèle normatif. La première entrée de chaque tableau, dite "repère chronologique", marque le début de la pratique dans le modèle visé ou, si cette indication n'était pas disponible, la date de publication du premier ouvrage définissant le modèle. La rubrique "visée» indique la direction spécifique donnée à l'action dans ce modèle. Sous le terme "références clés ", le tableau renvoie le lecteur à quelques ouvrages importants de définiteurs ou commentateurs du modèle.

Ces tableaux asseoient concrètement l'utilité du paradigme comme outil de connaissance de l'ensemble du champ de l'organisation communautaire en tant que pratique sociale. Les exemples choisis l'ont 


\section{TABLEAU 1}

Paradigme de l'organisation communautaire: orientations normatives et dimensions de la pratique

\begin{tabular}{|l|l|l|l|l|}
\hline $\begin{array}{l}\text { Dimension } \\
\text { de la pratique }\end{array}$ & Modèle normatif & Pression & Appropriation & Politisation \\
\hline $\begin{array}{l}\text { Contexte historique } \\
\text { Où? Quand? Dans quelles circonstances? }\end{array}$ & & & & \\
\hline $\begin{array}{l}\text { Idéologie } \\
\text { Conception de la société ? Conception du change- } \\
\text { ment social? }\end{array}$ & & & & \\
\hline $\begin{array}{l}\text { Problématique } \\
\text { Champ d'intervention? Situation-problème? Défini- } \\
\text { tion de la situation? }\end{array}$ & & & & \\
\hline $\begin{array}{l}\text { Population-cible } \\
\text { Qui ? Son rôle dans l'action? }\end{array}$ & & & \\
\hline $\begin{array}{l}\text { Agents(es) d'intervention } \\
\text { Qui agit? Comment se définissent les agents(es)? } \\
\text { Avec qui ? Face à qui ou contre qui ? }\end{array}$ & & & & \\
\hline $\begin{array}{l}\text { Processus d'intervention } \\
\text { Comment agit-on? Étapes? Moyens? }\end{array}$ & & & & \\
\hline $\begin{array}{l}\text { Impact } \\
\text { Résultats }\end{array}$ & & & & \\
\hline
\end{tabular}




\section{TABLEAU 2}

\section{Modèles d'intégration dans la littérature}

\begin{tabular}{|l|c|c|c|}
\hline Modele & \multicolumn{1}{|c|}{$\begin{array}{c}\text { Développement } \\
\text { communautaire } \\
\text { des Nations Unies }\end{array}$} & \multicolumn{1}{|c|}{$\begin{array}{c}\text { Organisation } \\
\text { communautaire } \\
\text { de Ross }\end{array}$} & $\begin{array}{c}\text { Animation sociale } \\
\text { au B.A.E.Q. }\end{array}$ \\
\hline Repère chronologique & 1955 & 1955 & 1963 \\
\hline Visée & $\begin{array}{l}\text { Associer les populations lo- } \\
\text { cales aux initiatives de déve- } \\
\text { loppement économique et } \\
\text { social planifiées par les États } \\
\text { récemment décolonisés, avec } \\
\text { l'aide d'experts internationaux }\end{array}$ & $\begin{array}{l}\text { Recréer une participation } \\
\text { communautaire consensuelle } \\
\text { autour de leaders locaux, dans } \\
\text { des communautés désorgani- } \\
\text { sées par l'industrialisation et } \\
\text { l'urbanisation }\end{array}$ & $\begin{array}{l}\text { Transformer la mentalité des } \\
\text { populations locales, pour les } \\
\text { amener à adhérer aux chan- } \\
\text { gements préconisés dans un } \\
\text { plan de développement éco- } \\
\text { nomique régional }\end{array}$ \\
\hline Références clés & $\begin{array}{l}\text { ONU, 1955; Salberg et Welsh- } \\
\text { Bonnard, 1970, pp. 6, 53-54, } \\
\text { 56-57, 118-129, 157-162, 188- } \\
192\end{array}$ & $\begin{array}{l}\text { Ross, 1955, 1958; Médard, } \\
1969, \text { pp. 128-132, 137-145; } \\
\text { Salberg et Welsh-Bonnard, } \\
1970, \text { pp. 57, 59, 63-65, 162- } \\
182\end{array}$ & $\begin{array}{l}\text { B.A.E.Q., 1966a, 1966b; Mo- } \\
\text { rency, 1966; Lebel, 1967; Si- } \\
\text { mard, 1978 }\end{array}$ \\
\hline
\end{tabular}

Autres exemples de modèles d'intégration, au Québec : animation sociale à la Télévision éducative du Québec (TÉVEC); participation des usagers à l'implantation des C.L.S.C.; organisation communautaire au Conseil du statut de la femme (C.S.F.); animation préventive de quartier au Service de réadaptation sociale (S.R.S.) de Québec (voir recension dans ce numéro). 
TABLEAU 3

Modèles de pression dans la littérature

\begin{tabular}{|l|c|c|c|}
\hline \multicolumn{1}{|c|}{ Modèle } & \multicolumn{1}{|c|}{$\begin{array}{c}\text { Syndicalisme } \\
\text { enseignant au Québec }\end{array}$} & \multicolumn{1}{|c|}{$\begin{array}{c}\text { Action libérale } \\
\text { radicale }\end{array}$} \\
\hline Repère chronologique & 1936 & 1939 & $\begin{array}{c}\text { Animation sociale } \\
\text { en milieu urbain } \\
\text { défavorisé }\end{array}$ \\
\hline Visée & $\begin{array}{l}\text { Organiser les salariés(es) et } \\
\text { créer un rapport de force } \\
\text { favorable dans la négociation } \\
\text { collective des conditions de } \\
\text { travail }\end{array}$ & $\begin{array}{l}\text { Acculer les possédants (les } \\
\text { have) à des compromis à } \\
\text { l'endroit des défavorisés (les } \\
\text { have not) par la force de la } \\
\text { mobilisation collective }\end{array}$ & $\begin{array}{l}\text { Susciter la participation des } \\
\text { citoyens des quartiers défa- } \\
\text { vorisés à la définition des } \\
\text { objectifs et des moyens du } \\
\text { développement urbain }\end{array}$ \\
\hline Références clés & $\begin{array}{l}\text { C.E.Q., 1985; C.S.N.-C.E.Q., } \\
1979, \text { pp. 101-102, 122, 133- } \\
134,151-152,168,174,185- \\
189,202-203,208\end{array}$ & $\begin{array}{l}\text { Alinsky, 1946, 1976; Médard, } \\
1970, \text { pp. 159-184 }\end{array}$ & $\begin{array}{l}\text { Blondin, 1965, 1967a, 1967b, } \\
1967 c ; \text { McGraw, 1978; Doré } \\
\text { et Plamondon, 1980 }\end{array}$ \\
\hline
\end{tabular}

Aut res exemples de modèles de pression, au Québec : syndicalisme à la C.S.N., F.T.Q. et C.S.D. ; comités de condition féminine dans les centrales syndicales. 


\section{TABLEAU 4}

\section{Modèles d'appropriation dans la littérature}

\begin{tabular}{|l|l|l|l|}
\hline \multicolumn{1}{|c|}{ Modèle } & \multicolumn{1}{|c|}{$\begin{array}{c}\text { Coopératives } \\
\text { d'habitation } \\
\text { au Québec }\end{array}$} & \multicolumn{1}{|c|}{$\begin{array}{c}\text { Entreprises } \\
\text { Communautaires } \\
\text { de P'Est du Québec }\end{array}$} & $\begin{array}{c}\text { Autogestion } \\
\text { ouvrière : Tricofil }\end{array}$ \\
\hline Repère chronologique & 1941 & 1970 & 1975 \\
\hline Visée & $\begin{array}{l}\text { Propriété et contrôle collec- } \\
\text { tifs de l'habitat }\end{array}$ & $\begin{array}{l}\text { Survie et développement } \\
\text { économique local, sous le } \\
\text { contrôle de la population } \\
\text { locale }\end{array}$ & $\begin{array}{l}\text { Contrôle des travailleurs et } \\
\text { travailleuses sur leur emploi } \\
\text { et leutreprise }\end{array}$ \\
\hline Références clés & $\begin{array}{l}\text { C.C.Q., 1968; Leduc, 1978; } \\
\text { Hurtubise, 1984 }\end{array}$ & $\begin{array}{l}\text { Roy, 1978; JAL, 1978; Sam- } \\
\text { son, 1978; Perron, 1978; Mi- } \\
\text { tis, 1978; Pelletier, 1978; Dra- } \\
\text { peau et Gagnon, 1982 }\end{array}$ & $\begin{array}{l}\text { Grand'Maison, 1975; Boucher, } \\
1982\end{array}$ \\
\hline
\end{tabular}

Autres exemples de modèles d'appropriation, au Québec : coopératives de consommation ; médias communautaires ; centres de santé et maisons de femmes; éco-développement (voir article dans ce numéro). 


\section{TABLEAU 5}

Modèles de politisation dans la littérature

\begin{tabular}{|l|l|l|l|}
\hline Indication & \multicolumn{1}{|c|}{ Conscientisation } & \multicolumn{1}{|c|}{$\begin{array}{c}\text { Action politique } \\
\text { syndicale à la C.S.N. }\end{array}$} & $\begin{array}{c}\text { Action politique } \\
\text { électorale au R.P.Q. }\end{array}$ \\
\hline Repère chronologique & 1962 & 1971 & 1977 \\
\hline Visée & $\begin{array}{l}\text { Développer la conscience cri- } \\
\text { tique et l'organisation des } \\
\text { classes opprimées, à travers } \\
\text { des luttes et des actions col- } \\
\text { lectives sur des problèmes } \\
\text { concrets et immédiats reliés } \\
\text { aux conditions de vie }\end{array}$ & $\begin{array}{l}\text { Susciter, parmi les syndi- } \\
\text { qués(es), un débat idéolo- } \\
\text { gique sur le projet et l'orga- } \\
\text { nisation politiques des travail- } \\
\text { leurs et travailleuses }\end{array}$ & $\begin{array}{l}\text { Faire déboucher les luttes des } \\
\text { comités de quartier sur un } \\
\text { parti municipal d'opposition } \\
\text { axé sur la défense des inté- } \\
\text { rêts urbains de la majorité }\end{array}$ \\
\hline Références clés & $\begin{array}{l}\text { Freire, 1973, 1974, 1978, 1980; } \\
\text { Ampleman et al., 1983; Bar- } \\
\text { nabé, 1985 }\end{array}$ & $\begin{array}{l}\text { C.S.N., 1971a, 1971b, 1973; } \\
\text { Favreau et L'Heureux, 1984 }\end{array}$ & $\begin{array}{l}\text { R.P.Q., 1977, 1984a, 1984b; } \\
\text { Racicot, 1980; Doré, 1983 }\end{array}$ \\
\hline
\end{tabular}

Autres exemples de modèles de politisation, au Québec : Front d'action politique des salariés (FRAP) et Comités d'action politique (CAP) de Montréal; Rassemblement des citoyens de Montréal (R.C.M.); groupes politiques marxistes-léninistes et trotskystes; Mouvement socialiste (M.S.); Regroupement pour le socialisme (R.P.S.). 
été en raison de leur représentativité à l'égard du modèle normatif visé, de leur influence passée ou présente en contexte québécois et du fait qu'ils ont donné lieu à une littérature facilement accessible. À cet égard, la bibliographie citée ne prétend pas être exhaustive. Elle ne retient que des ouvrages disponibles sur le marché. D'autres textes polycopiés et à diffusion restreinte présentent aussi beaucoup d'intérêt pour l'étude des modèles en organisation communautaire. Nous en tenons compte dans notre enseignement, à l'École de service social de I'Université Laval, en tâchant de rester à l'affût des pratiques nouvelles qui se diffusent dans des canaux artisanaux aux moyens limités, ou qui peuvent être suivies à la trace dans les dossiers de presse, à travers les reflets qu'en donnent les moyens de communication de masse.

Au bas de ces tableaux, d'autres exemples sont proposés et sont susceptibles, eux aussi, d'être bien documentés. Enfin, la bibliographie pourrait s'ouvrir à une littérature abondante sur l'histoire, les contextes sociologiques, les débats idéologiques et les mouvements sociaux dans lesquels s'inscrivent les pratiques en organisation communautaire, au Québec comme ailleurs.

Un autre intérêt du paradigme proposé est qu'il nous permet de comparer les courants qui se manifestent dans la littérature aux modèles normatifs qui s'actualisent dans la pratique d'un milieu donné. Nous tenterons, dans les lignes qui suivent, de l'utiliser pour dégager le tableau d'ensemble de la pratique de l'organisation communautaire au Québec, telle qu'elle ressort des données les plus récentes.

\section{Le paradigme et la pratique de l'organisation communautaire au Québec}

À partir d'enquêtes réalisées en 1978 (Doré et Larose, 1979) et en 1979 (La France, 1980), on peut respectivement situer dans le paradigme les orientations de pratique des organisateurs et organisatrices communautaires, soit dans leur ensemble au Québec et par catégorie d'organisme employeur, soit dans les C.L.S.C. du Montréal métropolitain.

Le tableau 6 est compilé à partir de l'enquête de 1978 du Regroupement des organisateurs et organisatrices communautaires du Québec (ROCQ). La concordance entre les catégories de l'enquête et le paradigme de l'organisation communautaire est donnée au bas du tableau. Il en ressort que les modèles de pression et de politisation se partagent chacun un tiers des organisateurs et organisatrices communautaires du Québec, alors que les modèles d'appropriation sont l'orientation normative de $18.2 \%$ d'entre eux et elles. Les modèles 


\section{TABLEAU 6}

Paradigme et modèles normatifs de pratique au Québec par catégorie d'organisme employeur (Enquête ROCQ, 1978)

\begin{tabular}{|c|c|c|c|c|c|c|c|c|c|c|}
\hline \multirow{2}{*}{$\begin{array}{l}\text { Modèle } \\
\text { normatif } * *\end{array}$ Organisme * } & \multicolumn{2}{|c|}{ O.R.A.S. } & \multicolumn{2}{|c|}{ A.O.G.P.G. } & \multicolumn{2}{|c|}{ O.B.E.P. } & \multicolumn{2}{|c|}{ S.C.G.P. } & \multicolumn{2}{|c|}{ Total } \\
\hline & $\mathbf{N}$ & $\%$ & $\mathbf{N}$ & $\%$ & $\mathbf{N}$ & $\%$ & $\mathbf{N}$ & $\%$ & $\mathbf{N}$ & $\%$ \\
\hline Intégration & 4 & 3.2 & 1 & 2.6 & 0 & 0.0 & 0 & 0.0 & 5 & 2.3 \\
\hline Pression & 47 & 37.9 & 12 & 30.8 & 10 & 50.0 & 5 & 13.5 & 74 & 33.6 \\
\hline Appropriation & 20 & 16.1 & 2 & 5.1 & 6 & 30.0 & 12 & 32.4 & 40 & 18.2 \\
\hline Politisation & 31 & 25.0 & 20 & 51.3 & 0 & 0.0 & 20 & 54.0 & 71 & 32.3 \\
\hline Hors paradigme & 22 & 17.7 & 4 & 10.3 & 4 & 20.0 & 0 & 0.0 & 30 & 13.6 \\
\hline Total & 124 & 100 & 39 & 100 & 20 & 100 & 37 & 100 & 220 & 100 \\
\hline Sans réponse & 5 & & 0 & & 3 & & 1 & & 9 & \\
\hline Échantillon & 129 & & 39 & & 23 & & 38 & & 229 & \\
\hline
\end{tabular}

* O.R.A.S. : Organismes du réseau des Affaires sociales

A.O.G.P.G.: Autres organismes gouvernementaux et para-gouvernementaux

O.B.E.P. : Organismes de bien-être privés

S.C.G.P. : Syndicats, coopératives et groupes populaires

** Selon les termes de l'enquête ROCQ, 1978 (Doré et Larose, 1979 : 92) :

Intégration: Participation aux établissements, animation technique et concertation des élites

Pression : Animation sociale et animation syndicale, sans perspective politique

Appropriation: Autogestion, sans perspective politique

Politisation : Conscientisation, direction politique marxiste-léniniste

Hors paradigme: Marketing et développement de services, coordination de groupes de bénévoles 
d'intégration n'occupent qu'une place marginale, ne ralliant que $2.3 \%$ des adhésions. Il est très important aussi de noter que $13.6 \%$ des répondants et répondantes qui avaient été identifiés comme organisateurs et organisatrices communautaires dans l'enquête ont une pratique de gestion des services sociaux plutôt qu'une pratique correspondant à la différence spécifique de l'organisation communautaire : intervention collective basée sur la participation active de ceux et celles qui vivent les situations-problèmes.

Le tableau 7 est compilé à partir d'une enquête réalisée en 1979 sur les organisateurs et organisatrices communautaires des C.L.S.C. du Montréal métropolitain, dans le cadre d'un cours de maîtrise du professeur Frédéric Lesemann de l'École de service social de l'Université de Montréal. La concordance entre la typologie des idéologies des organisateurs communautaires retenue dans cette enquête et le paradigme de l'organisation communautaire est donnée au bas du tableau. À noter qu'on ne trouve dans cette enquête aucun type correspondant au modèle d'appropriation de notre paradigme. La proportion des répondants et répondantes identifiés comme organisateurs et organisatrices communautaires, mais qui se situent en dehors du paradigme, est très élevée dans cette enquête, atteignant $30 \%$ des organisateurs et organisatrices communautaires du Montréal métropolitain.

Cette proportion se décompose elle-même en deux pourcentages qui méritent de retenir notre attention. Parmi ces $30 \%, 16.4 \%$ sont orientés vers l'organisation de services pour des "clients" et participent ainsi de la confusion largement explicitée plus haut entre gestion et organisation communautaire. Ce pourcentage est d'ailleurs légèrement inférieur à celui de ceux et celles qui donnent un contenu de gestion à l'organisation communautaire dans l'ensemble du réseau des Affaires sociales au Québec (17.7\%). Beaucoup plus frappant est le fait que $13.6 \%$ de ceux et celles qui se situent en dehors du paradigme se réclament d'une "idéologie de croissance personnelle». Selon les termes de l'enquête, dans ce type, "le travail de l'organisateur communautaire est de constituer des groupes, de fournir un cadre sécurisant d'intervention, d'utiliser les techniques modernes de la psychosociologie et de viser le mieux-être de chaque participant. La durée du groupe est temporaire». (La France, 1980: 20). Nous n'avons d'autre choix ici que de constater que des membres du personnel des C.L.S.C. du Montréal métropolitain, engagés comme organisateurs et organisatrices communautaires, appliquent à la place un des modèles du service social des groupes (groupwork). Cette situation n'est peut-être pas étrangère au fait que, dans les unités universitaires francophones de formation en travail social du Montréal métropolitain, le service 


\section{TABLEAU 7}

\section{Paradigme et modèles normatifs de pratique dans les C.L.S.C. du Montréal métropolitain (Enquête ESSUM, 1979)}

\begin{tabular}{|l|c|}
\hline Modèle normatif * & Pourcentage \\
\hline Intégration & 18.5 \\
Pression & 24.4 \\
Appropriation & 0.0 \\
Politisation & 24.7 \\
Hors paradigme & 30.0 \\
\hline Total & $97.6 * *$ \\
\hline
\end{tabular}

* Selon la typologie de l'enquête ESSUM, 1979 (La France, 1980 : pp. 20-22) : Intégration - type 2: Technique d'animation de groupes Pression - type 4 : Idéologie de participation et de conflit Appropriation : Aucun type correspondant à ce modèle normatif Politisation - type 5 : Mobilisation-conscientisation Hors paradigme - type 1 : Idéologie de croissance personnelle - type 3: Organisation de services

** Il s'agit d'une compilation de moyennes entre "accord complet " et " accord partiel»; d'où le total inférieur à $100 \%$.

social des groupes n'est plus systématiquement enseigné comme méthode distincte d'intervention en service social. Dans les circonstances, une clarification des contenus de formation et des définitions de poste semble s'imposer.

Le tableau 7 nous confirme donc la prédominance des modèles de pression et de politisation, et nous laisse voir une plus grande importance de ceux d'intégration dans les C.L.S.C. du Montréal métropolitain (18.5\%) que dans l'ensemble du réseau des Affaires sociales (3.2\%).

Une fois établies les orientations de pratique des organisateurs et organisatrices communautaires du Québec, il ne manquerait pas d'intérêt de leur comparer les intentions des organismes employeurs à leur endroit. Nous ne disposons pas de données systématiques en la matière, tout au plus d'indications concernant les plus importants 
employeurs institutionnels d'organisateurs et organisatrices communautaires au Québec: les centres locaux de services communautaires (C.L.S.C.).

Dans le présent numéro, l'article de Denis Bourque, organisateur communautaire en C.L.S.C., fait état des débats en cours au Ministère des affaires sociales et à la Fédération des C.L.S.C. du Québec concernant une conception de la distribution des services sociaux, présentée comme nouvelle et dite "approche communautaire». Au-delà des fioritures idéologiques, il ressort clairement que cette "approche communautaire", empruntée au rapport Barclay (Angleterre), s'inscrit en réalité dans un courant de retour sur la socialisation des risques sociaux à la base de l'État-Providence, pourtant gagnée de haute lutte comme un compromis historique entre la classe ouvrière et la classe capitaliste après la crise économique des années trente. La visée de cette approche consiste essentiellement à reporter sur des ressources privées gratuites ("réseaux primaires et communautaires") l'augmentation de la demande de services sociaux, elle-même occasionnée par l'actuelle crise économique.

Dans ce contexte, on peut prévoir que les organisateurs et organisatrices communautaires de C.L.S.C. recevront de leur employeur des mandats qui tendront à reproduire, en l'amplifiant, la situation observée jusqu'à maintenant.

Dans une logique gestionnaire braquée sur le court terme, des organisateurs et organisatrices communautaires continueront à subir des pressions pour réaliser des interventions qui sortent du paradigme ici défini. Pour arriver rapidement à des effets visibles en matière d'allègement des charges de cas et des listes d'attente, on leur demandera de se transformer en promoteurs et coordonnateurs d'agences bénévoles où les éléments actifs seront extérieurs aux collectivités qui vivent les situations-problèmes. Une autre modalité de ce schéma "assistancialiste", nouvelle version, sera l'incitation à multiplier les groupes d'entraide de courte durée, axés sur la psychologie des membres plutôt que vers les situations sociales d'où originent leurs problèmes.

Mais en même temps, on peut compter que la dimension d'aide et d'entraide que comportent en général les groupes d'organisation communautaire, comme corollaire de leur action sur le milieu (Deslauriers et Pouliot, 1982, pp. 36-44), ne passe pas inaperçue aux yeux de responsables d'établissements plus conscients des exigences d'une pratique réellement axée sur le changement social. En s'autorisant des beaux principes qui déguisent la visée réelle de l'«approche communautaire", ceux-ci sauront bien continuer à donner à leurs organisateurs 
et organisatrices communautaires la marge de manœuvre dont ils ont besoin pour développer des pratiques qui n'avaient pas attendu la "nouvelle» approche pour se mettre en œuvre.

\section{Notes}

1 Comme nous le verrons plus loin, selon la visée du modèle d'intervention en cause, il peut tout aussi bien s'agir d'une adaptation à des changements décidés par d'autres que d'une implication directe comme agent de changement.

2 Le développement des programmes de formation en organisation communautaire, dans les écoles de service social du Québec, suit de très près leur expansion aux États-Unis. Voir : J. MÉDARD, 1969, p. 225.

3 Pour une synthèse de ce débat dans la profession du service social entre l'organisation communautaire définie spécifiquement et l'organisation communautaire définie par assimilation à la gestion des services sociaux, voir : J. MÉDARD, 1969, pp. 214-225. Cet auteur parle d'un débat entre "processus" et "champ d'application" pour référer aux deux tendances en cause. À noter que l'ouvrage de Ross est paru sous ce titre dans sa première édition; il a été revu et publié à nouveau, en 1967, sous un titre légèrement modifié (voir Références bibliographiques).

4 Du grec "paradeigma " : exemple, modèle.

\section{Références bibliographiques}

Alinsky, S.D., Reveille for Radicals, Chicago, University of Chicago Press, 1946.

Alinsky, S.D., Manuel de l'animateur social, Paris, Seuil, 1976, 248p.

ampleman, G., G. Doré, L. Gaudreau, C. larose, C. Lebceuf et D. Ventelou, Pratiques de conscientisation: expériences, d'éducation populaire au Québec, Montréal, Nouvelle Optique, 1983.

BarNABÉ, J., "R.Q.S.E. du Nord: une pratique de conscientisation avec des femmes de classe populaire", Service social, vol. 34, nos 2-3, 1985.

Bureau d'aménagement de l'Est du Québec (B.A.E.Q.), "Participation", dans : Plan de développement, chapitre 1, cahier I, B.A.E.Q., 1966a: 27-33 et 38-39.

Bureau d'aménagement de l'Est du Québec (B.A.E.Q.), "Politique d'animation ", dans: Plan de développement, chapitre V, cahier 8, B.A.E.Q., 1966b: 132-143.

BLONDIN, M., "L'animation sociale en milieu urbain : une solution ", Recherches sociographiques, vol. VI, $n^{\circ} 3,1965: 283-304$.

BLondin, M., "La formation en service social", Bulletin de la Corporation des travailleurs sociaux professionnels de la province de Québec, no 15, décembre 1965-janvier 1966, pp. 7-8. 
BLONDIN, M., "Notes sur l'animation sociale en milieu urbain», Les Cahiers de I'I.C.E.A., numéro spécial sur l'Animation, no 4-5, 1967a : 51-71.

BLONDIN, M., "Quels changements apporte l'animation sociale?", Les Cahiers de I'I.C.E.A., op. cit., 1967b : 165-179.

BLONDIN, M., "Vie urbaine et animation sociale", dans: M.-A. LESSARD et J.-P. MONTMINY (éds), L'urbanisation de la société canadienne-française, Québec, Les Presses de l'Université Laval, 1967c : 111-119.

Boucher, P.-A., Tricofil, tel que vécu, Montréal, Éditions CIRIEC, 1982.

CARey-Bélanger, É., "Les débuts et le développement de la corporation (1960-1979)", Intervention, $\mathrm{n}^{\circ}$ 56, 1979: 5-25.

Centrale de l'enseignement du Québec (C.E.Q.), "Éducation syndicale», dans : Publications, Québec, C.E.Q., 1985. (Série de cahiers sur la méthodologie de l'action syndicale.)

Confédération des syndicats nationaux (C.S.N.), II n'y a plus d'avenir pour le Québec dans le système économique actuel, Montréal, C.S.N., 1971a.

Confédération des syndicats nationaux (C.S.N.), Ne comptons que sur nos propres moyens, Montréal, C.S.N., 1971b.

Confédération des syndicats nationaux (C.S.N.), Évaluation de la réflexion collective sur le document Ne comptons que sur nos propres moyens, Montréal, C.S.N., 1973.

C.S.N.-C.E.Q., Histoire du mouvement ouvrier au Québec (1825-1976), coédition C.S.N.-C.E.Q., 1979.

Conseil de la coopération du Québec (C.C.Q.), Les coopératives d'habitation au Québec, Québec, Rapport de la Commission de l'habitation du Conseil de la coopération du Québec, 1968.

DesLAURIERS, J.-P. et H. POulot, Les groupes populaires à Sherbrooke : pratique, financement et structure, Département de service social, Université de Sherbrooke, 1982.

DORÉ, G., "Le Rassemblement populaire de Québec", Relations, vol. 43, n 490, $1983: 123-125$.

DORÉ, G. et C. LAROSE, "L'organisation communautaire: pratique salariée d'animation des collectivités au Québec", Service social, vol. 28, $n^{\circ} 2-3$, 1979: 69-96.

Doré, G. et D. PlAmONDON, "Les pratiques urbaines d'opposition à Québec", Revue internationale d'action communautaire, 4/44, $1980: 120-128$.

Dunham, A., "Community organization for social work", dans : R.H. KURTZ (éd.), Social Work Year Book, New York, Russel Sage Foundation, vol. 7, 1943: 137-142.

Drapeau, M. et J.-G. Gagnon, Défaire la défaite! Histoire des luttes des paroisses du Bas-du-Fleuve, Rimouski, Édition SAIREQ, 1982.

Favreau, L. et P. L'heureux, Le projet de société de la C.S.N., de 1966 à aujourd'hui, Montréal, Centre de formation populaire, 1984.

FreIRE, P., "Éducation et conscientisation", dans : L'éducation : pratique de la liberté, Paris, Cerf, 1973 : 104-154. 
Freire, P., Pédagogie des opprimés suivi de Conscientisation et révolution, Paris, Maspero, 1974.

Freire, P., Lettres à la Guinée-Bissau sur l'alphabétisation, Paris, Maspero, 1978.

FreIRE, P., "Entretien avec Paulo Freire, 19 juin 1978, Genève", propos recueillis par Yvon Minvielle, Pourquoi?, no 151, $1980: 50-57$.

Grand'Maison, J., Une tentative d'autogestion, Montréal, Les Presses de l'Université de Montréal, 1975.

HARVEY, F., "Animation sociale et stratégie de développement régional dans l'Est du Québec", dans : Benoît LÉVESQUe (dir.), Animation sociale, entreprises communautaires et coopératives, Montréal, Albert Saint-Martin, 1978: 290-298.

HURtuBise, Y., "Orientations du développement des coopératives d'habitation au Québec", Service social, vol. 33, nº 1, 1984: 8-36.

JAL (les travailleurs du), "Le JAL", dans: B. LÉvESQUE, op. cit. : 63-73.

JEAN, B., "Animation et entreprise familiale agricole : le rôle des agronomes", id. : 225-236.

KURTZ, R.H. (éd.), Social Work Year Book, New York, National Association of Social Workers, 1943, pp. 137ss.

LA France, G., en collaboration avec Frédéric Lesemann, Modèles d'organisation communautaire: pratiques et idéologies dans les Centres locaux de services communautaires de la région de Montréal, École de service social, Université de Montréal, 1980.

Lamoureux, H., R. Mayer et J. Panet-Raymond, L'intervention communautaire, Montréal, Saint-Martin, 1984.

LAPLANTE, P., "L'organisation communautaire telle qu'on la pratique au Conseil des œuvres de Montréal ", Service social, vol. 11, $n^{\circ} 1,1962$ : 110-115.

LEBEL, J.-C., "L'animation sociale: expérience du B.A.E.Q.", Les Cahiers de I'I.C.E.A., numéro spécial sur la Participation de la collectivité à une planification économique, $n^{\circ} 3,1967: 57-75$.

LEDÚC, M., Les coopératives d'habitation, Montréal, Jour, 1978.

McGraw, D., Le développement des groupes populaires à Montréal, Montréal, Albert Saint-Martin, 1978.

MÉDARD, J., Communauté locale et organisation communautaire aux États-Unis, Paris, Armand Colin, 1969.

Meister, A., "Développement communautaire et planification", International Review of Community Development, $\mathrm{n}^{\circ}$ 9, 1962: 3-6.

Mitis (les travailleurs de la), "La Coopérative de production des maraîchers de la Mitis", dans: B. LéVESQUE, op. cit. : 93-102.

MorenCy, M.-A., Animation sociale : quelques éléments historiques de l'expérience du B.A.E.Q., Annexe technique du plan de développement, $\mathrm{n}^{\circ} 20$, 1966.

MORIN, C., "La méthode d'organisation communautaire dans le milieu canadienfrançais", Service social, vol. 11, $n^{\circ} 1,1962: 46-63$.

Organisation des Nations Unies (O.N.U.), Social Progress through Community Development, New York, O.N.U., 1955, $n^{\circ}$ de réf. 55 IV 18. 
Pelletier, B., "La scierie d'Esprit-Saint", dans : B. LÉvesque, op. cit. : 106-108.

Perron, G., "La Coopérative d'aménagement des ressources du Transcontinental dans le Témiscouata", dans : B. LÉVESQUE, op. cit. : 86-92.

RACicot, P., "Le Rassemblement populaire de Québec et les comités de citoyens", Revue internationale d'action communautaire, 4/44, 1980 : 129-133.

Rassemblement populaire de Québec (R.P.Q.), Manifeste, Québec, R.P.Q., 1977.

Rassemblement populaire de Québec (R.P.Q.), Québec, notre ville : programme du Rassemblement populaire, 1984a.

Rassemblement populaire de Québec (R.P.Q.), Le Rassemblement populaire de Québec : statuts, 1977, amendés en juin 1981, juin 1983 et novembre 1984b.

Ross, M.G. en collaboration avec B.W. LAPPIN, Community Organization: Theory, Principles and Practice, New York, Harper \& Row, $2^{\mathrm{e}}$ éd., 1967.

RothMAN, J., "Three models of community organization practice", dans : F.M. Cox et al. (éds), Strategies of Community Organization, Itasca (III.), Peacock, 1970: 20-36.

Roy, G., "L'animation sociale et la mise en place d'entreprises autogestionnaires : le point de vue d'un animateur", dans: B. LéveSQUE, op. cit. : 21-36.

SAMSON, M., "La cartonnerie de Cabano", dans: B. Lévesque, op. cit. : 74-77.

SAlBERG, J.-F. et S. WelSH-BonNARD, Action communautaire : une introduction, Paris, Éditions économie et humanisme, 1970.

SIMARD, J.-J., "De l'utopie à l'idéologie : planification, participation et pouvoir technocratique", dans : B. LÉVESQUE, op. cit. : 299-317. 